\title{
Integrated approach for efficient power consumption and resource allocation in MIMO-OFDMA
}

\author{
Archana B. ${ }^{1}$, T. P. Surekha ${ }^{2}$ \\ ${ }^{1}$ Department of Electronics and Communication Engineering, \\ GSSS Institute of Engineering and Technology for Women, Mysore, India \\ ${ }^{2}$ Department of Electronics and Communication Engineering, Vidyavardhaka College of Engineering, Mysore, India
}

\begin{tabular}{l} 
Article Info \\
\hline Article history: \\
Received Nov 2, 2018 \\
Revised Oct 18, 2019 \\
Accepted Nov 3, 2019 \\
\hline
\end{tabular}

\section{Keywords:}

Bit error rate (BER)

Convolution encoder

Parallel cancellation

Power consuption

Resource allocation

Signal to noise ratio (SNR)

Viterbi decoding

\begin{abstract}
The growing interest towards wireless communication advancement with smart devices has provided the desired throughput of wireless communication mechanisms. But, attaining high-speed data packets amenities is the biggest issue in different multimedia applications. Recently, OFDM has come up with the useful features for wireless communication however it faces interference issues at carrier level (intercarrier interferences). To resolve these interference issues in OFDM, various existing mechanisms were utilized cyclic prefix, but it leads to redundancy in transmitted data. Also, the transmission of this redundant data can take some more power and bandwidth. All these limitations factors can be removed from a parallel cancellation mechanism. The integration of parallel cancellation and Convolution Viterbi encoding and decoding in MIMOOFDMA will be an effective solution to have high data rate which also associations with the benefits of both the architectures of MIMO and OFDMA modulation approaches. This paper deals with this integrated mechanism for efficient resource allocation and power consumption. For performance analysis, MIMO-OFDMA system is analyzed with three different approaches likeMIMO-OFDM system without parallel cancellation (MIMO-OFDMA-WPC), MIMO-OFDMA System with parallel cancellation (MIMO-OFDMA-PC) and proposed IMO-OFDMA system with parallel cancellation and Convolution Viterbi encoding/decoding (pMIMO-OFDMAPC \&CVed) for $4 \mathrm{x} 4$ transmitter and receiver. Through performance analysis, it is found that the proposed system achieved better resource allocation (bandwidth) with high data rate by minimized BER rate and achieved least power consumption with least BER.
\end{abstract}

Copyright (0) 2020Institute of Advanced Engineering and Science. All rights reserved.

\section{Corresponding Author:}

Archana B.,

Department of Electronics and Communication Engineering,

GSSS Institute of Engineering and Technology for Women,

Mysore, India.

Email: archanab.research@gmail.com

\section{INTRODUCTION}

The growth in wireless communication with smart devices has enhanced the necessary throughput of wireless communication mechanisms [1]. But, attaining high-speed data packets amenities is the biggest issues various multimedia communications applications [2]. Among different existing wireless techniques, MIMO-OFDMA is considered an effective scheme to achieve this high data rate [3, 4]. Together these approaches can offer better performance and high data rate in wireless communication mechanisms [5]. In a recent trend, a huge number of high data rated devices were facing the problem of intersymbol interference (ISI) [6]. To perform the computation of the desired channel at the receiver end, channel 
estimation approaches can be used which improves the device capacity of OFDM system [7]. The channel estimation mechanisms mainly focus on the conceptuality of the favorable multiple path channels [8], but the existing techniques have introduced OFDM concepts with the real-time implementation of the multiple paths channels having a wide bandwidth that brings in OFDMA architecture [9, 10]. These multiple path communication channels acquire some propagation delays. Using conventional approaches, most of the elements of impulse response are of zero have some noise floor with least number of delayed path elements, and it idealizes that the multiple path channels exhibit the sparse architecture [11-16]. Thus, no such mechanisms exist for computation of sparse data. Hence, the traditional computational techniques do not use this communication channel having a sparse signal. However, with available MIMOOFDMA transceiver system, it is the biggest concern to achieve minimized BER for the receive data signal generated by the device. This manuscript gives an integrated approach for resource allocation and power consumption in a MIMO-OFDMA system having 4x4 transmitter and receiver by considering BER and SNR with better signal quality. The categorization of this paper is performed as Background of MIMO-OFDMA system with existing techniques, Problem description, System model and implementation (Section 2), Performance analysis (Section 3) and Conclusion (Section 4).

- The background

This section deals with the review of existing techniques that considers MIMO-OFDMA. The previous work of Archana and Sureka [17] discussed a research survey stating the current state of the art in research domain subjected to MIMO-OFDMA system for efficient communication. To mitigate the intercell interference Perez et al. [18] have presented a self-organizing algorithm which enhances system-level performance. The [18] limits with intercell communication.

Similarly, Alsohaily et al. [19] analyzed the user access for multi-radio which also lags with intercell communication. The resource allocation in smart devices is considered by Huang et al. [20] and introduced a protocol based algorithm by using Nash equilibrium which brings better gain and sum rate. A computationally efficient optimal algorithm is presented in Xiao et al. [21] which improves the quality of service (QoS) through efficient resource allocation, and it is not compared with any existing technique. The OFDM systems are lacking with the high peak to average ratio (PAPR), and this issue is addressed in Ait-Saadi et al. [22]. The considerable reason behind high PAPR is due to non-linear distortions generated by power amplifiers. Among various existing techniques, partial transmit sequence is a significant technique which helps in minimizing the PAPR. However, achieving a considerable level of PAPR reduction is the biggest concern as it leads to higher computational complexity. Thus, [22] introduced an algorithm of self-adaptive multi population differential evolution which brings a low-cost system for PAPR optimization. The efficient scheduling mechanism for resource allocation in MIMO is found in Cao et al. [23] where the two-step method is used to bring the balance to computational complexity and resource allocation. The previous work of Archana and Sureka [24] presented a compressive sensing based channel estimator for MIMO-OFDMA. With the numerical outcome of [24] suggests that the system was a low-cost system to have better MIMO-OFDMA system. The work towards performance analysis of MIMO-OFDMA system in energy efficiency perspective is found in Singal and Kedia [25] where different mechanisms for antenna selection were considered, i.e., per-subcarrier and bulk selection, etc. with hardware architecture. On analyzing both the mechanisms, it has been found that bulk selection of antenna has come up with energy efficient than the per-subcarrier selection of antenna mechanism. From the above surveys, it was observed that very rare works were incorporated with the minimization of BER, resource allocation, and power consumption.

- The Problem Statement

The significant features of OFDM made it as an efficient modulation scheme. However, it exhibits some interference at carriers, i.e., inter-carrier interference. TO overcome these interferences some of the existing techniques utilized cyclic prefix but it leads to redundancy issue in transmitted data. Further transmission of this redundant data can utilize more power and bandwidth during transmission. This redundancy in data can be eliminated by using parallel cancellation technique. The research practices in recent years have suggested that many real-time multi-path channels with a broad bandwidth may lead to sparse in architecture. During this, the communication channel of multipath collects some propagation delays. The conventional approaches provide impulse response elements of zero under the noisy condition with few delayed path elements which indicate sparse architecture of multipath channel. Hence no conventional mechanism performs the estimation of sparse data where these mechanisms ignore the sparse signal to minimize the BER of received data. Thus, there is a need of a system to be developed which can consider the above pitfalls and come up with energy efficient and better resource allocation in MIMO OFDMA. 


\section{SYSTEM MODEL AND IMPLEMENTATION}

In order to overcome the above-discussed problems, the MIMO-OFDMA system is analyzed with three different approaches likeMIMO-OFDMA system without parallel cancellation (MIMO-OFDMAWPC), MIMO-OFDMA System with parallel cancellation (MIMO-OFDMA-PC) and proposed MIMOOFDMA system with parallel cancellation and Convolution Viterbi encoding/decoding (pMIMO-OFDMAPC \&CVed) for 4x4 transmitter and receiver. In the proposed system, MIMO-OFDMA concept is considered to minimize BER ratio under high noise condition. The MIMO works on the basis of optimal binary search tree (OBST) scheme. The transmitter side of OFDMA uses IFFT operation while the receiver side uses FFT operation. However, to overcome Inter-Carrier Interference issues few transmitters data is processed by using IFFT operation in a MIMO system. In the proposed system, instead of the parallel operation of IFFT and FFT, all the transmitter data are processed through FFT operation at transmitter side while IFFT operation at the receiver side. The inter-changing of both FFT and IFFT at transmitter and receiver the zero value of BER can be achieved at $5 \mathrm{~dB}$ in traditional MIMO-OFDMA system while in proposed MIMO-OFDMA system zero BER can be achieved at $-5 \mathrm{~dB}$. In this paper also, a method of Forward error correction (FEC) and detection approaches like Convolutional encoding and Viterbi decoding utilized before the modulation of transmitted data and after demodulation of the received data respectively. The FEC mechanism also provides an additional protection layer for data in terms of detection and correction of errors in received data by the $1 \mathrm{~dB}$ improvement.

Further to augment OFDM systems, some notations on OBST-OFDMA were analyzed with the 4x4 OBST-OFDM codeword matrix which offers diversity order 2 having a coding rate of 1 . For the complex transmission, this orthogonality can't be achieved with a code rate of 1 during the transmit diversity is exceeding 2. There exist some of the studies to offer a framework for non-orthogonal complex transmission with a coding rate of 1 and having diversity order equal to the number of transmit antennas. Thus, a 4x 4 OBST-OFDM system is considered with a non-orthogonal transmission matrix, the coding rate of 1 , and transmission diversity of 4 . Also, the system of orthogonal transmission matrix is analyzed with a code rate of 0.5 . The Following Figure 1 gives the architectural model of the proposed system.

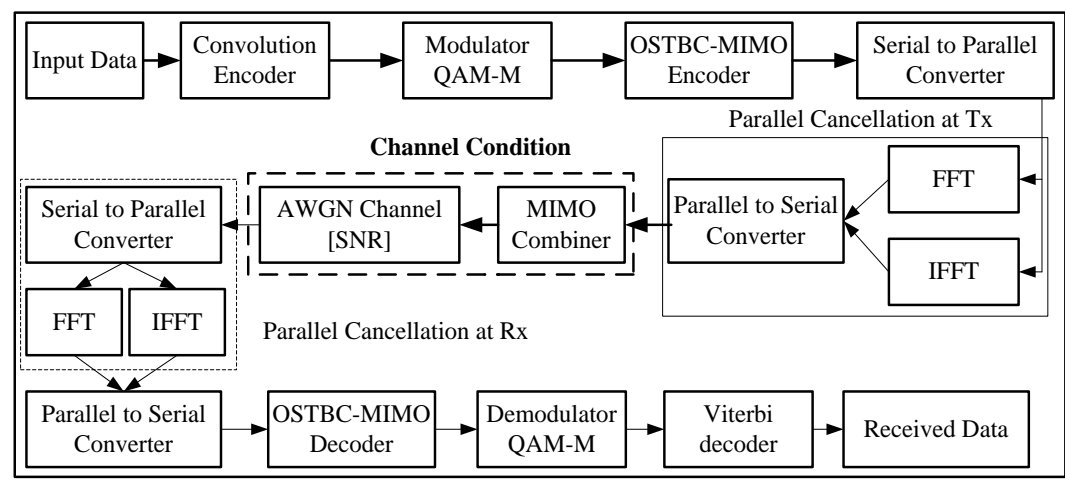

Figure 1. Architectural model of the proposed system

In the proposed system an image is considered and is then converted into a binary format. On the same binary data pre-processing operations can be performed to get good quality of input signal. Further, the convolution encoding approach is adapted over the input signal. The same signal is modulated by using the QPSK modulation technique. This modulated signal is encoded using the OSTBC encoding technique depending upon the number of transmitters are used in this framework. At the transmitter end, the OFDM modulation will be done along with the parallel cancellation scheme. Then the signal is converted time domain to frequency domain. This signal is transmitted through the communication channel. The channel includes the MIMO channel along with AWGN noise. This signal to noise ratio is adjusted using signal range. Then it is transmitted. The received signal is demodulated using the OFDM Demodulating scheme along with parallel cancellation method. These received and demodulated signal is applied with OSTBC combining scheme depending upon the number of receiver devices are used in this framework. Then, this signal is demodulated using the QPSK demodulating method. The Viterbi decoding scheme is used to filter all the noise present in the received signal. In the end, the received data is converted to the original format. Then, the performance analysis is performed by considering bit error rate (BER). The BER is compared with three different approaches likeMIMO-OFDMA system without parallel cancellation (MIMO-OFDMA-WPC), MIMO-OFDMA System with parallel cancellation (MIMO-OFDMA- 
PC) and proposed MIMO-OFDMA system with parallel cancellation and Convolution Viterbi encoding/decoding (pMIMO-OFDMA-PC \&CVed) for 4x4 transmitter and receiver. The flow diagram given in Figure 2 represents the implementation of the algorithm in the system model.

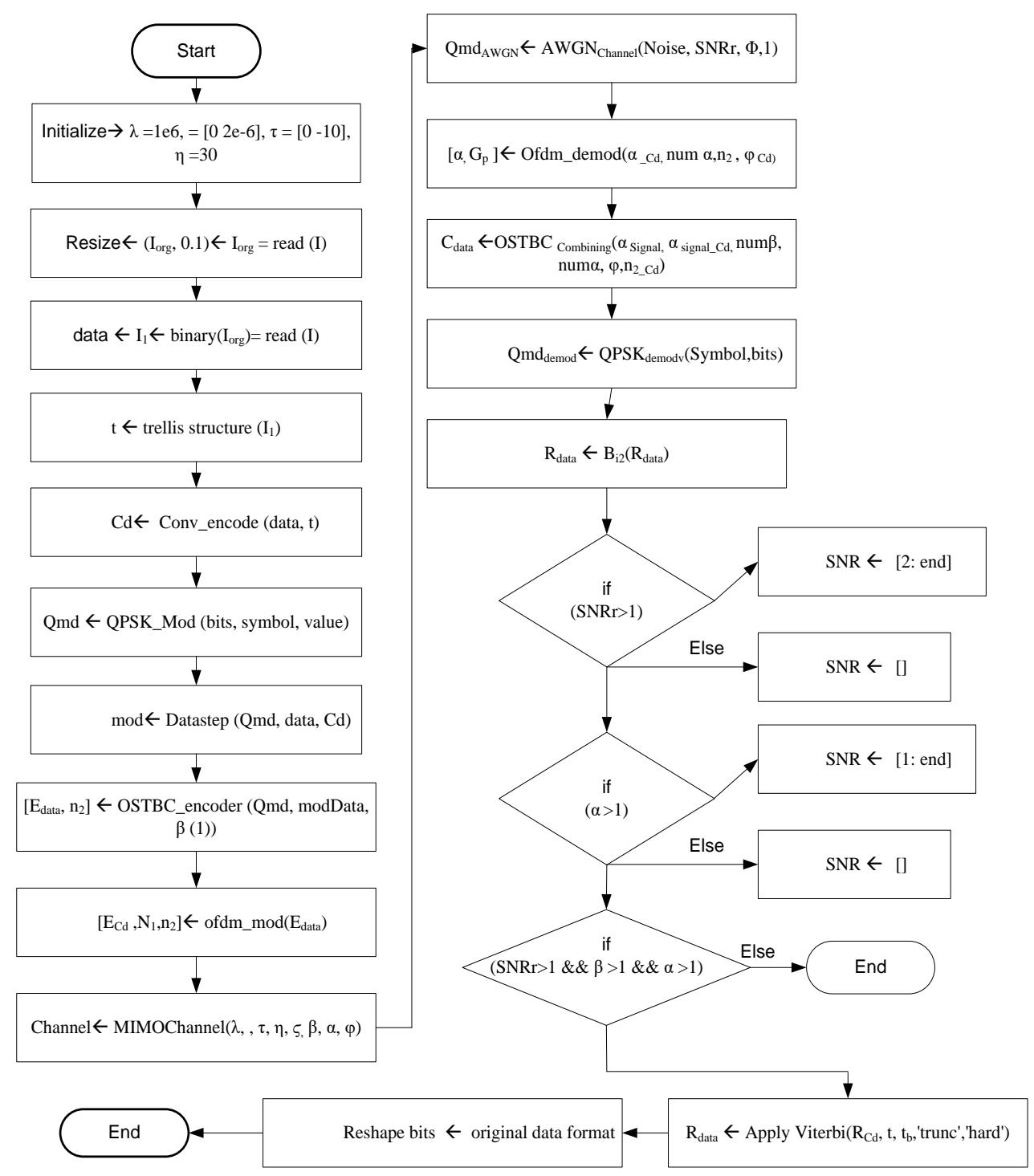

Figure 2. Flow representation of the implemented algorithm

During first step of the algorithm, the initialization of sampling rate $(\lambda=1 \mathrm{e} 6)$, path delay $\left(\delta=\left[\begin{array}{ll}0 & 2 \mathrm{e}-6\end{array}\right]\right)$, average path gain $\left(\tau=\left[\begin{array}{ll}0 & -10\end{array}\right)\right.$, maximum Doppler shift $(\eta=30)$ is performed. Later, an image from the disk is selected and is resized with a multiplication factor of 0.1 . The resized image is converted into binary form and is stored as data. Using MATLAB, trellis structure ( $\mathrm{t}$ ) of a stored binary form of data is formed for convolution encoder which provides every possibility of input to the encoder subjected with both outputs and state transition of the encoder having states of binary form 00, 01, 10 and 11 which yields 2-bit output from 1-bit input.

Further, considering both the factors data and the convolution encoder is applied which gives a convoluted data $(\mathrm{Cd})$. The modulation scheme QPSK is applied to the signal to yield the modulated signal, and then the encoding is performed by using orthogonal space-time block code (OSTBC) encoder block that encodes the input symbol sequence using OSTBC. The use of OSTBC along with MIMO helps to yield high SNR and low BER. The OSTBC can be adapted with a system having feedback to the transmitter from the receiver. Later, the signal is demodulated by using the QPSK demodulation approach. For the same 
signal, the SNR values will be calculated and then apply the MIMO-OFDMA technique by considering original data, SNR value, transmission range, and receiver range. Later, the Viterbi decoding mechanism is adapted to filter the noise in the received signal. Finally, the received signal is converted/reshaped into the original format. Table 1 exhbits the description of the notations used in the proposed implementation algorithm as discussed in Figure 2.

\begin{tabular}{lll}
\multicolumn{3}{c}{ Table 1 Description of notations } \\
\hline SI.No & Notation & Description \\
\hline 1 & I & Input image \\
2 & I1 & Binary image \\
3 & Cd & Convoluted data \\
4 & Qmd & QPSK modulated data \\
5 & $\alpha$ & Receiver range \\
6 & $\beta$ & Transmitter range \\
7 & SNRr & SNR range \\
8 & $\lambda$ & Sampling rate \\
9 & $\delta$ & Path delay \\
10 & $\tau$ & average path gain \\
11 & $\eta$ & maximum Doppler shift \\
12 & $\varphi$ & Path gain \\
13 & 5 & Spatial Correlation \\
14 & $\Phi$ & Signal power \\
\hline
\end{tabular}

\section{PERFORMANCE ANALYSIS}

The proposed integrated mechanism for efficient resource allocation and power consumption in MIMO-OFDMA system of $4 \times 4$ transmitter and receiver is in terms of BER and SNR with better signal quality under noisy conditions. The simulation of the method is performed by using MATLAB. The performance is analyzed by comparing with three different approaches likeMIMO-OFDMA system without parallel cancellation (MIMO-OFDMA-WPC), MIMO-OFDMA System with parallel cancellation (MIMO-OFDMA-PC) and proposed MIMO-OFDMA system with parallel cancellation and Convolution Viterbi encoding/decoding (pMIMO-OFDMA-PC \&CVed) for $4 \times 4$ transmitter and receiver. In the proposed pMIMO-OFDMA-PC \&CVedsystem, the parallel operation of FFT and IFFT were replaced at both transmitter and receiver which help to reduce BER. The proposed system introduced Forward error correction (FEC) and detection mechanisms like convolutional encoding before modulating the transmitted data and Viterbi decoding after demodulating the received data. This significance of the FEC mechanism is that it offers an additional protective layer for the data in terms of detection and correction of errors in received data by the $1 \mathrm{db}$ improvement. The following section follows with the outcomes accomplished with respect to different approaches for communication. The comparative analysis is considered with 4-transmitter and 4-receiver for all the different approaches likeMIMO-OFDMA system without parallel cancellation (MIMO-OFDMA-WPC), MIMO-OFDMA System with parallel cancellation (MIMO-OFDMA-PC) and proposed MIMO-OFDMA system with parallel cancellation and Convolution Viterbi encoding/decoding (pMIMO-OFDMA-PC \&CVed) for $4 \times 4$ transmitter and receiver. The following Figure 3 gives the outcomes attained from MATLAB simulation for all the approaches at different SNR values.

The Figures 4-6 represent the plot of different approaches like MIMO-OFDMA system without parallel cancellation (MIMO-OFDMA-WPC), MIMO-OFDMA System with parallel cancellation (MIMOOFDMA-PC) and proposed MIMO-OFDMA system with parallel cancellation and Convolution Viterbi encoding/decoding (pMIMO-OFDMA-PC \&CVed) for $4 \times 4$ transmitter and receiver. From Figures 4-6 it is clearly observed that the BER increases with a decrease in the Signal to Noise Ratio (SNR) value. The SNR with least value indicates the signal is having more unwanted noise while least value of BER indicates the least error occurred during signal transmission. In general sense, the BER is reciprocal to SNR. Thus, the comparison of all these approaches gives that found that the MIMO-OFDMA system without parallel cancellation (MIMO-OFDMA-WPC) has highest BER than other approaches having MIMOOFDMA System with parallel cancellation (MIMO-OFDMA-PC) and proposed MIMO-OFDMA system with parallel cancellation and Convolution Viterbi encoding/decoding (pMIMO-OFDMA-PC \&CVed). This indicates that the error rate in MIMO-OFDMA-WPC is high as it does not reduce the unwanted noise. In comparison with the MIMO-OFDMA-PC and pMIMO-OFDMA-PC \&CVed, the proposed system has the least error rate than another approach. Thus it can be said that the proposed pMIMO-OFDMA-PC \&CVedsystem in terms of power and resource allocation (bandwidth) is achieved with high data rate by minimized BER rate and also with least BER the power consumption is reduced. 


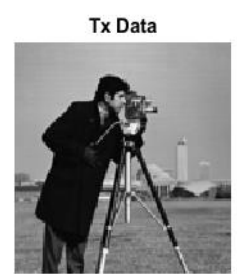

Tx Data

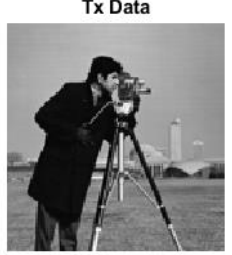

Tx Data

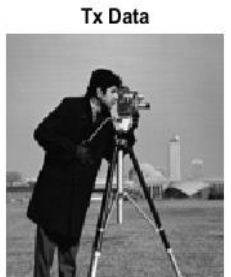

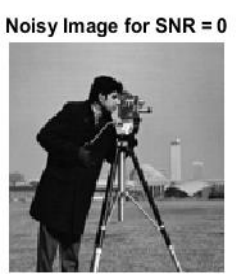

Noisy Image for SNR $=$

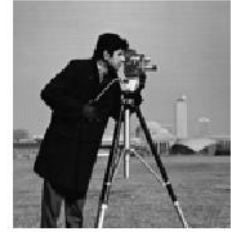

Noisy Image for SNR $=30$

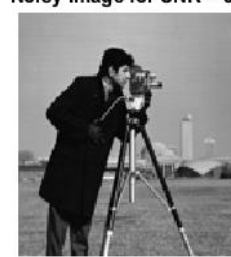

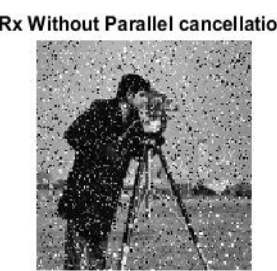

Rx Without Parallel cancellation

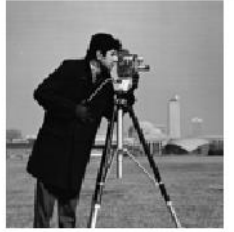

Rx Without Parallel cancellation

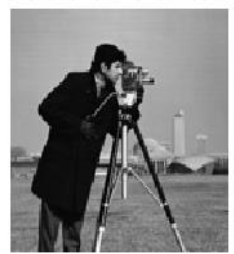

Rx Image With OFDM PC

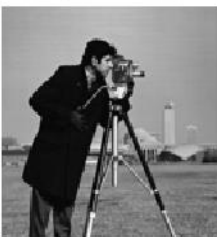

Rx Image With OFDM PC

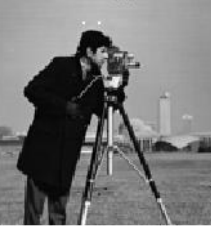

Rx Image With OFDM PC

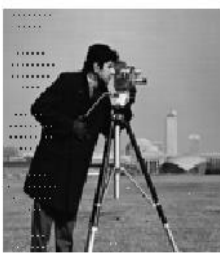

Rx Image With OFDM PC CV

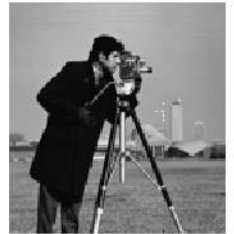

Rx Image With OFDM PC CV

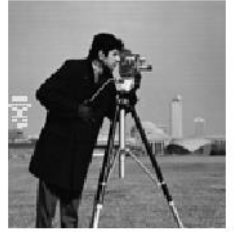

Rx Image With OFDM PC CV

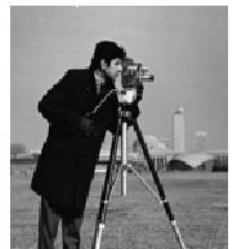

Figure 3. 4 x 4 transmitter and receiver for data

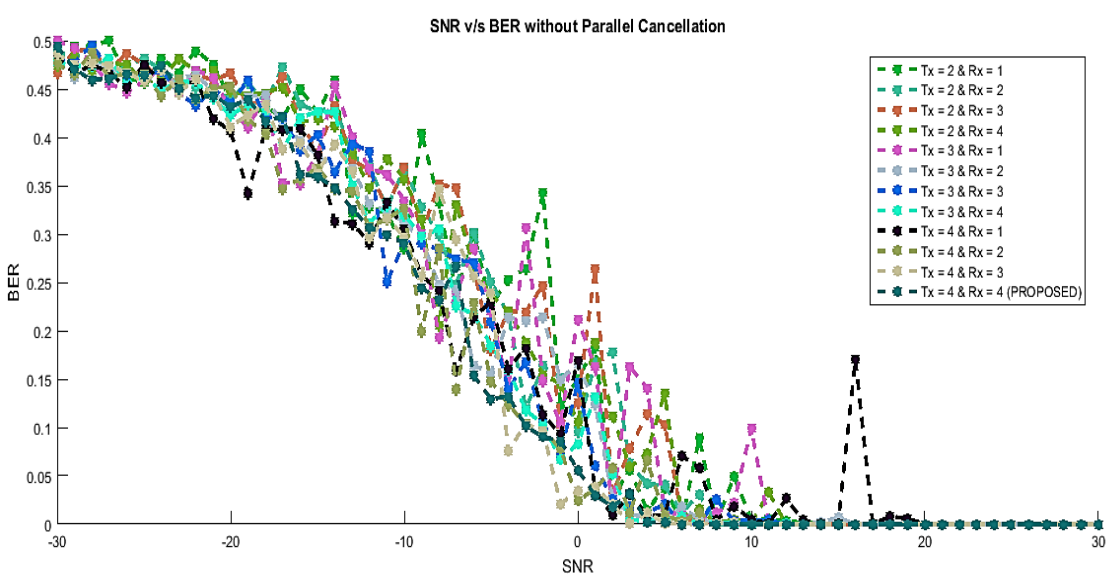

Figure 4 SNR vs. BER without parallel cancellation

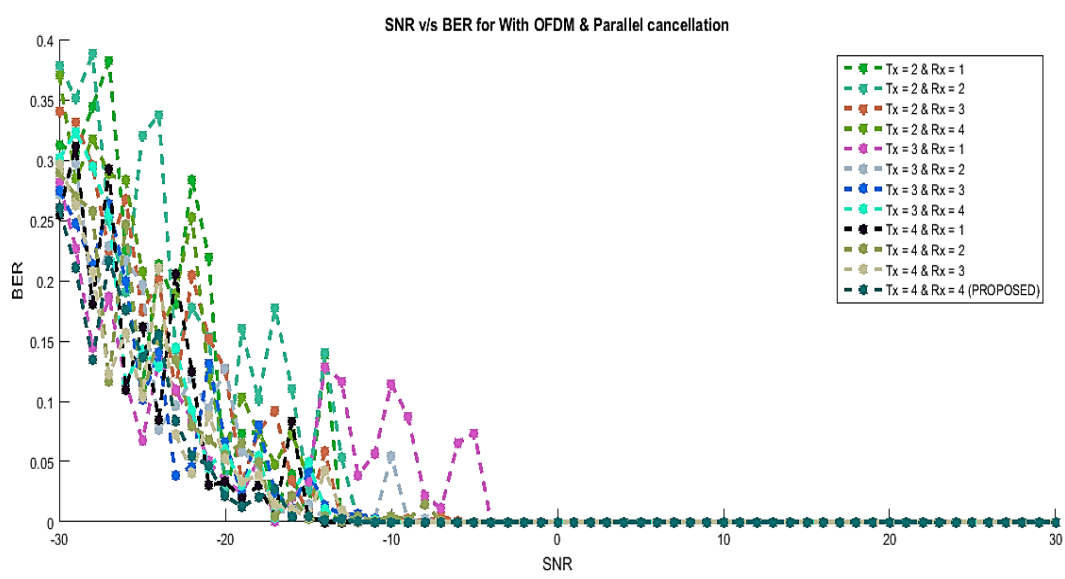

Figure 5 SNR vs. BER with OFDM \&parallel cancellation 


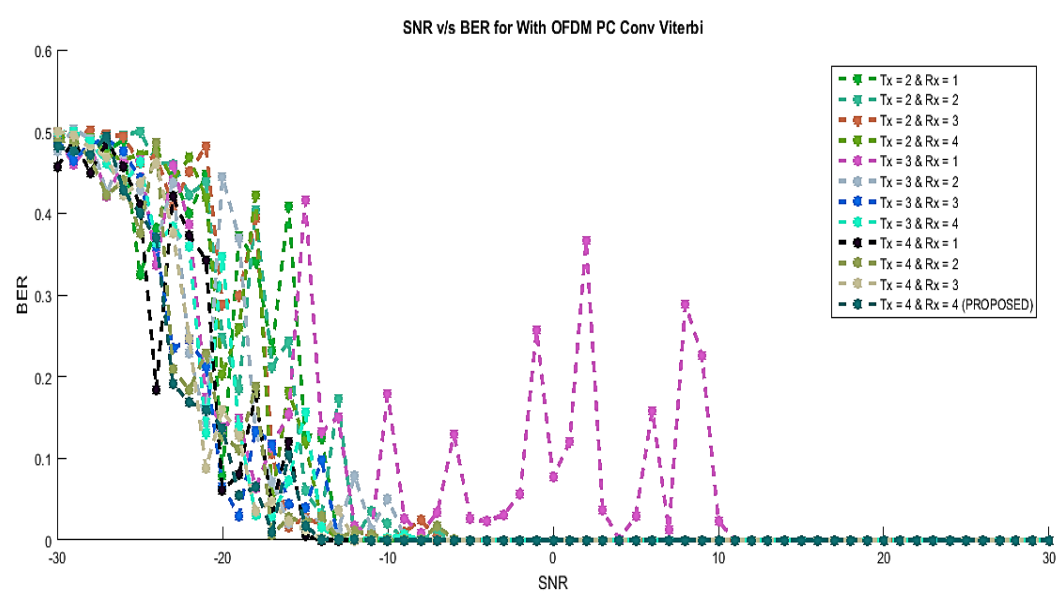

Figure 6 SNR vs. BER with OFDM-PC convolution viterbi

\section{CONCLUSION}

This paper presents a MIMO-OFDMA system with parallel cancellation and Convolution Viterbi encoding/decoding (pMIMO-OFDMA-PC \&CVed) system of 4x4 transmitter and receiver to achieve better resource allocation and power consumption in terms of BER and SNR with better signal quality. Through this pMIMO-OFDMA-PC \&CVedsystem, computational complexity and cost of the system are minimized. The proposed pMIMO-OFDMA-PC \&CVedsystem is having a combination of parallel cancellation, and Viterbi encoding and decoding (Proposed system) achieved less BER than MIMO-OFDMA system without parallel cancellation (MIMO-OFDMA-WPC), MIMO-OFDMA System with parallel cancellation (MIMOOFDMA-PC) approach. From this, it can be concluded that the system attained high data rate transmission by minimizing the BER rate and power consumption. The proposed system can be considered in future research to meet security issues of MIMO-OFDMA having parallel cancellation and Viterbi encoding or decoding approach.

\section{REFERENCES}

[1] Checko, Aleksandra, et al., "Cloud RAN for mobile networks-A technology overview," IEEE Communications surveys \& tutorials, vol. 17, no. 1, pp:405-426, 2015.

[2] Khalifa, Tarek, Kshirasagar Naik, and Amiya Nayak, "A survey of communication protocols for automatic meter reading applications," IEEE Communications Surveys \& Tutorials, vol. 13, no. 2, pp:168-182, 2011.

[3] Feng, Daquan, et al., "A survey of energy-efficient wireless communications," IEEE Communications Surveys \& Tutorials, vol. 15, no. 1, pp:167-178, 2013.

[4] Harjula, Ilkka, et al., "Practical issues in the combining of MIMO techniques and RoF in OFDM/A systems," Proceedings of the 7th WSEAS International Conference on Electronics, Hardware, Wireless, and Optical Conference. 2008.

[5] Nosratinia, Aria, Todd E. Hunter, and AhmadrezaHedayat, "Cooperative communication in wireless networks," IEEE communications Magazine, vol. 42, no. 10, pp:74-80, 2004

[6] Olwal, Thomas O., Karim Djouani, and Anish M. Kurien, "A survey of resource management toward 5G radio access networks," IEEE Communications Surveys \& Tutorials, vol. 18, no. 3, pp:1656-1686, 2016.

[7] Akyildiz, Ian F., Dario Pompili, and Tommaso Melodia, "State-of-the-art in protocol research for underwater acoustic sensor networks," Proceedings of the 1st ACM international workshop on Underwater networks. ACM, 2006.

[8] Gkelias, Athanasios, and Kin K. Leung, "Multiple antenna techniques for wireless mesh networks," Wireless Mesh Networks. Springer, Boston, MA, 277-307, 2008.

[9] Rawat, Priyanka, et al., "Wireless sensor networks: a survey on recent developments and potential synergies," The Journal of supercomputing, vol. 68, no. 1, pp:1-48, 2014.

[10] Hughes, Laurie, Xinheng Wang, and Tao Chen, "A review of protocol implementations and energy efficient crosslayer design for wireless body area networks," Sensors, vol. 12, no. 11, pp:14730-14773, 2012.

[11] Tse, David, and Pramod Viswanath, "Fundamentals of wireless communication," Cambridge university press, 2005.

[12] Bajwa, Waheed U., et al., "Compressed channel sensing: A new approach to estimating sparse multipath channels," Proceedings of the IEEE, vol. 98, no. 6, pp:1058-1076, 2010.

[13] Wheeler, Heather E., Kaanan P. Shah, Jonathon Brenner, Tzintzuni Garcia, Keston Aquino-Michaels, Nancy J. Cox, Dan L. Nicolae, Hae Kyung Im, and GTEx Consortium. "Survey of the heritability and sparse architecture of gene expression traits across human tissues," PLoS genetics, vol. 12, no. 11, e1006423, 2016. 
[14] Wheeler, Heather E., Kaanan P. Shah, Jonathon Brenner, Tzintzuni Garcia, Keston Aquino-Michaels, Nancy J. Cox, Dan L. Nicolae, Hae Kyung Im, and GTEx Consortium. "Survey of the heritability and sparse architecture of gene expression traits across human tissues." PLoS genetics, vol. 12, no. 11 e1006423, 2016.

[15] Yang, Xi, and Byrav Ramamurthy, "Sparse regeneration in translucent wavelength-routed optical networks: Architecture, network design and wavelength routing." Photonic network communications 10, no. 1 (2005): 39-53.

[16] Olshausen, Bruno A., Phil Sallee, and Michael S. Lewicki. "Learning sparse image codes using a wavelet pyramid architecture," In Advances in neural information processing systems, pp. 887-893. 2001.

[17] Archana B. and T. P. Surekha, "Resource Allocation in LTE: An Extensive Review on Methods, Challenges, and Future Scope", Communications on Applied Electronics (CAE) - ISSN: 2394-4714 Foundation of Computer Science FCS, New York, USA Volume 3- No.2, October 2015.

[18] Lopez-Perez, D. Chu, X., Vasilakos, A.V., Claussen, H.2014, "Power Minimization Based Resource Allocation for Interference Mitigation in OFDMA Femtocell Networks," Selected Areas in Communications, IEEE Journal, vol.32, no.2, pp.333-344

[19] Alsohaily, A., Sousa, E.S., "On the Utilization of Multi- Mode User Equipment in Multi-Radio Access Technology Cellular Communication Systems," Access, IEEE, vol. 3, pp.787-792, 2015.

[20] Huang, J., Yin, Y., Zhao, Y., Duan, Q., Wang, W., Yu, S., "A Game-Theoretic Resource Allocation Approach for IntercellDevice-to-Device Communications in Cellular Networks," Emerging Topics in Computing, IEEE Transactions, no. 99, pp.1-1, 2014.

[21] Xiao, X., Tao, X., Lu, J., "Energy-Efficient Resource Allocation in LTE-Based MIMO-OFDMA Systems with User Rate Constraints," Vehicular Technology, IEEE Transactions on, vol. 64, no. 1, pp:185-197, 2015.

[22] Ait-Saadi, Hocine, Jean-Yves Chouinard, and AbderrazakGuessoum. "A PAPR Reduction for OFDM Signals Based on Self-Adaptive Multipopulation DE algorithm," International Journal of Electrical and Computer Engineering (IJECE), vol. 7, no. 5, pp:2651-2660, 2017.

[23] H. Cao, J. Cai, A. Alfa and Z. Zhao, "Efficient resource allocation scheduling for MIMO-OFDMA-CR downlink systems," 2016 8th International Conference on Wireless Communications \& Signal Processing (WCSP), Yangzhou, pp. 1-5, 2016.

[24] Archana, B., and T. P. Surekha. "A Compressive Sensing Based Channel Estimator and Detection System for MIMO-OFDMA System." In Proceedings of the Computational Methods in Systems and Software, pp. 22-31. Springer, Cham, 2018.

[25] Singal, Anuj, and Deepak Kedia. "Performance Analysis of Antenna Selection Techniques in MIMOOFDM System with Hardware Impairments: Energy Efficiency Perspective," International Journal of Electrical and Computer Engineering (IJECE), vol. 8, no. 4, pp:2272-2279, 2018.

\section{BIOGRAPHIES OF AUTHORS}

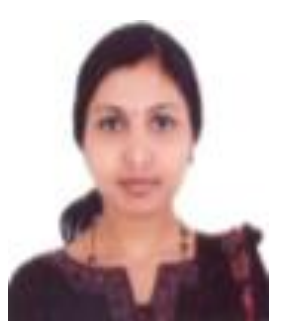

Archana Bworking as Assistant Professor,Department of Electronics and Communication Engineering,GSSS Institute of Engineering \& Technology for Women, Mysuru, Karnataka, India. She is pursuing Ph.D in Wireless Communication, VTU Resaerch Centre under the guidance of Dr.T P Surekha. She has published 2 international Conference paper.

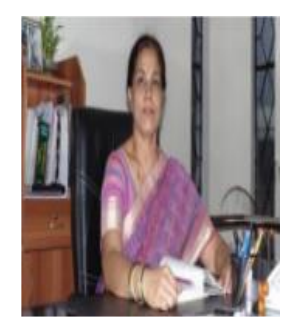

Dr. T P Surekha, Professor, Department of Electronics and Communication Engineering, Vidyavardhaka College of Engineering, Mysuru, Karnataka, India. She has completed her PhD in Communication Systems from Visvesvaraya Technological University, Belagavi, Karnataka, India. She has around more than 25 years of teaching experiemce. She has published 15 national/international journals and 29 national/international conferences papers. 